\title{
Cloning of Perilipin 2 Gene and Investigating its Expression Level in Porcine Longissimus Muscle
}

\author{
Yang $\mathrm{M}^{\sharp 1}$, Dong $\mathrm{K}^{\sharp 2}$, Shu $\mathrm{X}^{1}$, Li $\mathrm{W}^{1}$, Huang $\mathrm{Y}^{1}$, Pan $\mathrm{H}^{* 1}$, and Zhao $\mathrm{S}^{{ }^{*}}$
}

${ }^{1}$ Yunnan Key Laboratory of Animal Nutrition and Feed Science, Yunnan Agricultural University, Kunming 650201, China

${ }^{2}$ Faculty of Food Science and Technology, Yunnan Agricultural University, Kunming 650201, China

\#These authors contributed equally to this work and regarded as the co-first author

*Corresponding author(s): Pan H, Zhao S, Yunnan Key Laboratory of Animal Nutrition and Feed Science, Yunnan Agricultural University, Kunming 650201, China, E-mail: 286589395@qq.com, zhaosm2009@126. com

Citation: Yang M, Dong K, Shu X, Li W, Huang Y, et al. (2017) Cloning of Perilipin 2 Gene and Investigating its Expression Level in Porcine Longissimus Muscle. J Vet Sci Ani Husb 5(1): 106. doi: 10.15744/23489790.5.106

Received Date: October 28, 2016 Accepted Date: February 25, 2017 Published Date: February 28, 2017

\begin{abstract}
PLIN2 belongs to one member of PAT (Perilipin, Adipophilin and Tip47) family, which plays an important role in regulating lipid storage and could be regarded as a candidate gene for intramuscular fat deposition in pigs. This study tried to clone the coding domain sequence (CDS) of PLIN2 gene, compare the nucleotide acids and deduced amino acids sequence, physiological characteristics, structure and the expression level between Wujin (fatty breed) and Landrace (lean breed) pigs. The results showed that the mutation of nucleotide acids led to the mutation of deduced amino acids between two pig breeds. The molecular weight and isoelectric point value as well as the phosphorylation sites were changed, but the N-Glycosylation sites, hydrophobic characteristics and homology Swiss model kept the same. The expression level of PLIN2 gene in Wujin pigs was significantly higher than Landrace. These results suggested that the changes of nucleotide acids sequence of PLIN2 gene could not change the structure and did not affect the function. The expression of PLIN2 gene could play important role in the intramuscular fat (IMF) content.

Keywords: Perilipin 2 gene; Nucleotide acids; Amino acids; Expression level

List of Abbreviations : IMF: Intramuscular fat; PLIN2: Perilipin 2; ADPH: Adipophilin; ADRP: Adipose Difference-Related Protein; CDS: Coding Domain Sequence; RNA: Ribonucleic acids; RT: Reverse Transcription; PLINs: Lipid Droplet-Associated Proteins
\end{abstract}

\section{Introduction}

In pig production, intramuscular fat (IMF) content is one of the determinant factors of meat quality characteristics such as tenderness, juiciness and flavor level [1]. IMF content varies in different pig breeds [2]. Particularly, IMF content is higher in the Chinese local pigs than in other commercial pigs [3-5]. The Wujin pig is one of the Chinese local pigs, which is regarded as fatty genotype [6,7]. The Landrace pig is considered a lean breed. The IMF content is significantly different between these two breeds [8]. Therefore, the underlying mechanism of fat deposition in fatty and lean pigs could be elucidated using these two pig models $[8]$.

Many efforts have been carried out to identify key factors and genes affecting IMF [9]. Porcine perilipin 2 (PLIN2) is also called adipophilin (ADPH), adipose difference-related protein (ADRP). PLIN2 genetic variability has been more intensively investigated in Chinese, Korean breeds, characterized by higher fat deposition, and compared with European pig breeds, more intensively selected for lean meat [10-12]. The biological role and mapping localization suggested that porcine PLIN2 could be a promising functional and positional candidate gene for intramuscular fat deposition in pigs [13-16]. However, little information is available about the nucleotides and amino acids sequence and the expression level between Chinese and European pig breeds.

Therefore, the objective of this research was to clone the CDS (coding domain sequence) sequence, compare the similarity of nucleotide and deduced amino acids sequence between Wujin and Landrace pigs and detect the expression level of gene as well as analyze the association between the expression level and intramuscular fat in Wujin pigs. 


\section{Materials and Methods}

All experiment procedures were performed according to the Guide for Animal Care and Use of Laboratory Animals in the Institutional Animal Care and Use Committee of Yunnan Agricultural University. The experimental protocol was approved by the Department Animal Ethics Committee of Yunnan Agricultural University.

\section{Animal and samples}

The feeding and diet of the used pigs has been detailed by Zhao et al. [8]. Briefly, six Wujin and six Landrace pigs were used. All pigs had free access to water from nipple drinkers. The diets were not isonitrogenous or isoenergetic because the growth rate of the two breeds was so divergentand were provided ad libitum. When the pigs reached $100 \mathrm{~kg}$ body weight, they were transported to Yunnan Agricultural Center Meats Laboratory and slaughtered after electrical stunning. Longissimus muscle samples from the last ribs were collected from every animal. Samples were cut into small pieces, frozen in liquid nitrogen, and stored at $-75^{\circ} \mathrm{C}$ for later analyses.

\section{Total RNA extraction and reverse transcript}

Total RNA was extracted from all the samples using the Total RNA Extraction Kit (Invitrogen, America) as per the manufacturer's instructions. Total RNA concentration was quantified by measuring the absorbance at $260 \mathrm{~nm}$ in a photometer (Eppendorf Biophotometer). Ratios of absorption $(260 / 280 \mathrm{~nm})$ of all preparations were between 1.8 and 2.0. Aliquots of RNA samples were subjected to electrophoresis through a $1.4 \%$ agarose formaldehyde gel to verify their integrity.

Reverse transcription was performed using the RNA $(2 \mu \mathrm{g})$ described above in a final volume of $25 \mu \mathrm{L}$ containing 10 units of MMLV reverse transcriptase (Promega, Belgium), $1 \mathrm{mM}$ dNTP mixture (Promega, Belgium), 40 units of recombinant RNasin ribonuclease inhibitor (Promega, Belgium) and $0.5 \mu \mathrm{L}$ of oligo (dT) 18 (Promega, Belgium) in sterilized water and buffer supplied by the manufacturer. After incubation at $42{ }^{\circ} \mathrm{C}$ for $60 \mathrm{~min}$, the mixture was heat treated at $95{ }^{\circ} \mathrm{C}$ for 5 min. cDNA samples were kept in $-20^{\circ} \mathrm{C}$ for detection.

\section{cDNA clone}

The reverse transcription (RT) reaction mix $(2 \mu \mathrm{L})$ was used for PCR in a final volume of $25 \mu \mathrm{L}$ containing $1.5 \mathrm{mM}$ $\mathrm{MgCl} 2,200 \mu \mathrm{M}$ dNTP, $1.5 \mathrm{IU}$ Taq polymerase and $50 \mathrm{pmol}$ of the forward and reverse primers. The primers was F:5'GAAGAAACATGGCATCCGTTGCTGT-3',R:5'-GCTGCTTCTCCACTTGGGAATTGTC-3'. The primers were designed based on the porcine sequence (Accession No. NM_214200.1). The fragment length of the amplified PCR product was 1412bp.

Aliquots of the PCR products were analyzed by electrophoresis in a 1.5\% agarose gels. The gels were stained with ethidium bromide and photographed with a digital camera. The final products were cloned into pGEM-T Easy vector (Takara, Japan).

\section{Plasmid extraction, diagnostic digestion and sequencing}

White colonies were picked up with a sterile wooden toothpick and were inoculated into tubes with $3 \mathrm{~mL}$ of agar containing 100 $\mathrm{mg} / \mathrm{mL}$ ampicillin. Tubes were incubated on a shaker at $37^{\circ} \mathrm{C}$ and $100 \mathrm{rpm}$ for $12-18 \mathrm{~h}$. Plasmids were extracted using a Qiagen Plasmid Purification Mini Kit (Qiagen) according to the manufacturer recommendations. Five $\mu \mathrm{L}$ of plasmid, $12 \mu \mathrm{L}$ of DNase free water, $1 \mu \mathrm{L}$ of either $10 \mathrm{U} / \mu \mathrm{L}$ EcoRI or $10 \mathrm{U} / \mu \mathrm{L}$ XbaI and $2 \mu \mathrm{L}$ of the respective $10 \mathrm{X}$ reaction buffer were added to a final volume of $20 \mu \mathrm{L}$ and incubated for $1 \mathrm{~h}$ at $37^{\circ} \mathrm{C}$. As a control, $3 \mu \mathrm{L}$ of uncut plasmid and $17 \mu \mathrm{L}$ of DNase free water were added to a final volume of $20 \mu \mathrm{L}$ and incubated for $1 \mathrm{~h}$ at $37^{\circ} \mathrm{C}$. The vector containing the insert had $5 \mathrm{EcoRI}$ and 1 XbaI restriction enzyme sites. Following diagnostic digestion, digestion products were loaded on a $1 \%$ agarose gel with ethidium bromide.Following verification of the presence of insert, purified plasmids were sequenced (Takara, Japan).

\section{Real-time RT-PCR}

Real-time PCR was performed to quantify the expression abundance of PLIN2 mRNA. The $25 \mu$ PCR mixture contained $12.5 \mu \mathrm{l}$ of $2 \times \mathrm{iQ}^{\mathrm{m}}$ SYBR Green Supermix, $0.5 \mu \mathrm{l}(10 \mathrm{mM})$ of each primer and $1 \mu \mathrm{l}$ of cDNA. Mixtures were incubated in an iCyler iQ Realtime Detection system (Bio-Rad, America) programmed to conduct 40 cycles $\left(95{ }^{\circ} \mathrm{C}\right.$ for $15 \mathrm{~s}, 65{ }^{\circ} \mathrm{C}$ for $35 \mathrm{~s}$ and $72{ }^{\circ} \mathrm{C}$ for $65 \mathrm{~s}$ ). The primers of PLIN2 gene were F: 5'- TCTATCTGTCCTGGGTGG -3', R: 5' - ATCGTGGATGTTCTGTGG -3'. The control gene was 18S rRNA. The Primers of 18S rRNA were F: 5'-CTGCCTCCTTGGATGTG-3', R: 5'-GCGGCTTTGGTGACTCTA-3'. The quantification of the transcripts was performed using a standard curve with 10 -fold serial of dilution of cDNA. Melt curve was conducted to ensure that only a single PCR product was amplified. Samples were assayed and each experiment was repeated at least twice. Control reactions (without template or Taq enzyme) were set for each sample.

\section{Bioinformatics, sequence Alignment, and statistical Analysis}

The cDNA sequence prediction was conducted using GenScan software (http://genes.mit.edu/ GENSCAN.html). The protein prediction and analysis were performed using the Conserved Domain Architecture Retrieval Tool of BLAST at the National Center 
for Biotechnology Information (NCBI) server(http://blast.ncbi.nlm.nih.gov/), the ClustalW software (http://www.ebi.ac.uk/Tools/ clustalw2/ index.html) and DNAstar software.

Isoelectric point and molecular weight ( $\mathrm{pI} / \mathrm{Mw}$ ) of deduced amino acids sequence was analyzed in compute $\mathrm{pI} / \mathrm{Mw}$ (http://web. expasy.org/compute_pi/). NetPhos 2.0 Server (http://www.cbs.dtu.dk/services/NetPhos/) was used to predict the phosphorylation sites [17]. ProtScale allows to compute and represent the profile produced by any amino acid scale on a selected protein (http://web. expasy.org/protscale/). The NetNglyc server predicts N-Glycosylation sites in human proteins using artificial neural networks that examine the sequence context of Asn-Xaa-Ser/Thr sequins (http://www.cbs.dtu.dk/services/NetNGlyc/) [18]. Swiss-Model (http:// swissmodel.expasy.org/) was employed to homology modeling of three ovine proteins, and RasMol V2.7.5 was used to visualize the PDB files generated by Swiss-model [19].

Comparison of gene expression between Wujin and Landrace pigs was accomplished by Student's t-test. The statistical analyses were performed using the SAS software system (SAS Inst. Inc., Cary, NC).

\section{Results}

\section{Cloning and Sequencing of Genes}

To clone and sequence the PLIN2 gene for Wujin and Landrace pigs, reverse transcriptase PCR was used to amplify the PLIN2 gene. The amplified sequence length of PLIN2 was 1, 412bp (Figure 1). Sequencing of the resulting cDNA showed the complete coding sequence of PLIN2 to be 1,038 bp. Sequences from Landrace pigs kept the same as the GenBank (accession numbers, NM_214200.1). Sequences from Wujin pigs were deposited in the GenBank database (accession numbers, JN616387). The PLIN2 gene between these two breeds is highly conserved. Nucleotide sequences analysis showed that the Wujin pigs. The nucleic acids sequence from Wujin pigs was $99 \%$ identical to the Landrace (Figure 2).

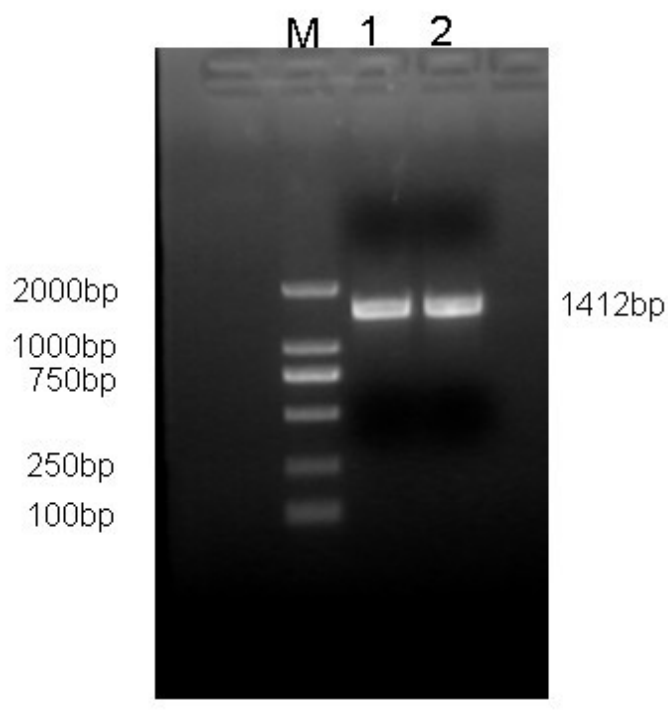

Figure 1: Amplification fragment of PLIN2 gene

M: DL2000marker; 1,2: PCR product from Wujin and Landrace pigs

Computer generation of the AA sequence from the nucleic acid sequence revealed open reading frames of 459 AA. The deduced amino acid sequences of PLIN2 showed 99\% identity between Wujin and Landrace pigs (Figure 3).

The differences in nucleotide and amino acid sequences between the two breeds were given as following. The base changes at nucleotides 80 ( $\mathrm{G}$ to A), 315 (A to G), 399 (A to G), 904 (C to T), 936 (A to G), 992 (A to G), 998 (G to A) and 1304 (G to A). The nucleotides changes at 80, 904, 992, 998 and 1304 resulted in the amino acids mutation at 27 (G to D), 302 (A to C), 331 (N to $S$ ), 333 ( $\mathrm{R}$ to $\mathrm{Q}$ ) and 435 ( $\mathrm{R}$ to $\mathrm{H}$ ).

\section{Physical and chemical characteristics of deduced proteins}

The molecular weight and theoretical pI of the deduced amino acid sequence of PLIN2 in Wujin and Landrace pigs was approximately 50179.76 and $50248.90 \mathrm{Da}$ and a of 6.18 and 7.08 respectively, predicted by theCompute pl/Mw program. The predicted phosphorylation sites showed that the PLIN2 protein in Wujing and Landraces pigs had Ser: 17, Thr: 11, Tyr: 7 and Ser: 16, Thr: 12, Tyr: 7 respectively, which have been shown in Figure 4. The difference of Ser and Thr phosphorylation sites exited in 35 and 433. The PLIN2 had the same N-glycosylated sites in Wujin and Landrace pigs. Four potential N-glycosylated sites were indicated (Figure 5). PLIN2 protein from Wujin and Landrace pigs belongs to hydrophilic protein (Figure 6). 


\begin{tabular}{|c|c|c|}
\hline PLIN2-W & ATGGCATCCGTTGCTGTTGAATCACAACCGAGTGTGGTGACAAGGGTGGCCAACCTACCC & 6 \\
\hline PLIN2-L & ATGGCATCCGTTGCTGTTGAATCACAACCGAGTGTGGTGACAAGGGTGGCCAACCTACCC & \\
\hline PLIN2-W & TTGGTGAGCTCCACGTATdA ACCTTGTCTCCTCGGCTTATATCAGTACAAAGGATCAGTAT & 120 \\
\hline PLIN2-L & TTGGTGAGCTCCACGTATdGCCTGTCTCCTCGGCTTATATCAGTACAAAGGATCAGTAT & \\
\hline PLIN2-W & ССCTACTTGAAGTCTCTGTGTGAGATGGCAGAGAAGGGCGTCAAGACCATCACCTCCGTG & \\
\hline PLIN2-L & CCCTACTTGAAGTCTCTGTGTGAGATGGCAGAGAAGGGCGTCAAGACCATCACCTCCGTG & \\
\hline PLIN2-W & GCCATGTCCGGTGCTCTCCCTATCATCCAGAAGCTAGAGCCTCAGATTGCCATTGCCAAC & \\
\hline PLIN2-L & GCCATGTCCGGTGCTCTCCCTATCATCCAGAAGCTAGAGCCTCAGATTGCCATTGCCAAC & \\
\hline PLIN2-W & ACTTACGCCTGTAAGGGACTAGACAGAATTGAGGAGAAGCTGCCTATTCTGAATCAGCCA & \\
\hline PLIN2-L & ACTTACGCCTGTAAGGGACTAGACAGAATTGAGGAGAAGCTGCCTATTCTGAATCAGCCA & \\
\hline PLIN2-W & ACAAACCAGGTTGIGFCCAATGCTAAAGGGGCTGTGACTGGGGCAAAAGATGCTATGACG & 30 \\
\hline PLIN2-L & ACAAACCAGGTTGAAFCCAATGCTAAAGGGGCTGTGACTGGGGCAAAAGATGCTATGACG & \\
\hline PLIN2-W & ACTACTGTGACTGGGGCCAAGGATTGTGTGGCCAGCAdGA & 14 \\
\hline PLIN2-L & ACTACTGTGACTGGGGCCAAGGATTGTGTGGCCAGCA A ATCACTGAGGTGGTGGACAAG & $42 C$ \\
\hline PLIN2-W & ACCAAAGAAGCAGTGACCGGAAGTGTGGAAAAGACCAAGTCTGTGGTTAATGGAAGCATT & \\
\hline PLIN2-L & ACCAAAGAAGCAGTGACCGGAAGTGTGGAAAAGACCAAGTCTGTGGTTAATGGAAGCATT & \\
\hline PLIN2-W & AACACTGTCCTGGGAAGTCGGATGATGCAGCTGGTGAGCAGTGGAGTAGAAAAAGCATTC & \\
\hline PLIN2-L & AACACTGTCCTGGGAAGTCGGATGATGCAGCTGGTGAGCAGTGGAGTAGAAAAAGCATTC & \\
\hline PLIN2-W & ACCAAATCAGAGCTGCTGGTAGACCAGTACCTCCCTCTCACTGAAGAAGAACTAGAAAAA & \\
\hline PLIN2-L & ACCAAATCAGAGCTGCTGGTAGACCAGTACCTCCCTCTCACTGAAGAAGAACTAGAAAAA & \\
\hline PLIN2-W & GAAGCCAAAAAAGTGGAAGGATTTGATATGGTTCAGAAGCCAAGTTATTATATTAGACTG & 000 \\
\hline PLIN2-L & GAAGCCAAAAAAGTGGAAGGATTTGATATGGTTCAGAAGCCAAGTTATTATATTAGACTG & 66 \\
\hline PLIN2-W & GGATCCCTGTCCACCAAGCTCCGCTCACGGGCCTACCAGCAGGCCCTCACCAGGGTTAAA & \\
\hline PLIN2-L & GGATCCCTGTCCACCAAGCTCCGCTCACGGGCCTACCAGCAGGCCCTCACCAGGGTTAAA & \\
\hline PLIN2-W & GAAGTCAAGCAAAAAAGCCAGGAGACCATTTCTCAGCTCCATTCCACTGTCAACCTGATT & \\
\hline PLIN2-L & GAAGTCAAGCAAAAAAGCCAGGAGACCATTTCTCAGCTCCATTCCACTGTCAACCTGATT & \\
\hline PLIN2-W & GAATTTGCCAGGAAGAATGTGCATAATGCCAACCAGAAAATTCAAGGCACTCAGGATAAG & 84 \\
\hline PLIN2-L & GAATTTGCCAGGAAGAATGTGCATAATGCCAACCAGAAAATTCAAGGCACTCAGGATAAG & \\
\hline PLIN2-W & СTCTATCTGTCCTGGGTGGAATGGAAGAGAAGCATTGGCTATGATGACACAGATGAATCC & 30 \\
\hline PLIN2-L & СTCTATCTGTCCTGGGTGGAATGGAAGAGAAGCATTGGCTATGATGACACAGATGAATCC & 90 \\
\hline PLIN2-W & CAфПGTGCTGAGCATATAGAGTCACGTACTCTGG GATTGCCCGCAACCTGACTCAGCAG & \\
\hline PLIN2-L & CA@GTGCTGAGCATATAGAGTCACGTACTCTGG AATTGCCCGCAACCTGACTCAGCAG & 96 \\
\hline PLIN2-W & СTCCAGACCACGTGCCACACCCTCGTGTCCAGCATCDAA GGGTTACCACAGAACATCCAC & 102 \\
\hline PLIN2-L & CTCCAGACCACGTGCCACACCCTCGTGTCCAA A ATCDGAGGGTTACCACAGAACATCCAC & 10 \\
\hline PLIN2-W & GATCAGGCCAACCACTTGGGGGTGATGGCTGGCGACATCTACTCAGTGTTTCACAATGCT & 00 \\
\hline PLIN2-L & GATCAGGCCAACCACTTGGGGGTGATGGCTGGCGACATCTACTCAGTGTTTCACAATGCT & 108 \\
\hline PLIN2-W & TCCTCCTTTAAGGAAATGTCTGATGGCCTCCTCAGTTCCAGCAAG & \\
\hline PLIN2-L & TCCTCCTTTAAGGAAATGTCTGATGGCCTCCTCAGTTCCAGCAAGGGGCAGCTGCAGAAA & 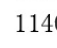 \\
\hline PLIN2-W & ATGAAGGAGTCTTTAGATGATGTGATGGATTATCTTGTTAACAACACGCCCCTCAACTGG & \\
\hline PLIN2-L & ATGAAGGAGTCTTTAGATGATGTGATGGATTATCTTGTTAACAACACGCCCCTCAACTGG & 1200 \\
\hline PLIN2-W & CTGGTAGGTCCCTTTTATCCTCAACTGACCGAGTCTCAGGATGC & \\
\hline PLIN2-L & CTGGTAGGTCCCTTTTATCCTCAACTGACCGAGTCTCAGGATGCTCAGTCCCGGGGTGCA & 1260 \\
\hline PLIN2-W & GAGAACACAACGAGCCCAGAGACCCAGCAACCTGAGACAAAAdACATTAAACCTGCCCCT & \\
\hline PLIN2-L & GAGAACACAACGAGCCCAGAGACCCAGCAACCTGAGACAAAAdGCATTAAACCTGCCCCT & \\
\hline & AGLAGISLA IS & \\
\hline LIN2-L & GCCAGCAGTGCATGGGGCAGCCAGTCAGGTGACACGTCCTGTACTGTTGCAACCTGCTAG & \\
\hline
\end{tabular}

Figure 2: Comparison of Nucleotide acids sequence between Wujin and Landrace pigs Sequence indicates Landrace pigs and Sus was Wujin pigs 


\begin{tabular}{|c|c|c|}
\hline PLIN2-W & MASVAVESQPSVVTRVANLPLVSST DLLVSSAY ISTKDQYPYLKSLCEMAEKGVKTITSV & 60 \\
\hline PLIN2-L & MASVAVESQPSVVTRVANLPLVSST GLVSSAY ISTKDQYPYLKSLCEMAEKGVKTITSV & 60 \\
\hline PLIN2-W & AMSGALPIIQKLEPQIA IANTYACKGLDRIEEKLPILNQPTNQVVANAKGAVTGAKDAMT & 120 \\
\hline PLIN2-L & AMSGALPIIQKLEPQIAIANTYACKGLDRIEEKLPILNQPTNQVVANAKGAVTGAKDAMT & 120 \\
\hline PLIN2-W & TTVTGAKDCVASTITEVVDKTKEAVTGSVEKTKSVVNGSINTVLGSRMMQLVSSGVEKAF & 180 \\
\hline PLIN2-L & TTVTGAKDCVAST ITEVVDKTKEAVTGSVEKTKSVVNGS INTVLGSRMMQLVSSGVEKAF & 180 \\
\hline PLIN2-W & TKSELLVDQYLPLTEEELEKEAKKVEGFDMVQKPS YY IRLGSLSTKLRSRAYQQALTRVK & 240 \\
\hline PLIN2-L & TKSELLVDQYLPLTEEELEKEAKKVEGFDMVQKPSYYIRLGSLSTKLRSRAYQQALTRVK & 240 \\
\hline PLIN2-W & EVKQKSQETISQLHSTVNLIEFARKNVHNANQKIQGTQDKLYLSWVEWKRSIGYDDTDES & 300 \\
\hline PLIN2-L & EVKQKSQETISQLHSTVNLIEFARKNVHNANQKIQGTQDKLYLSWVEWKRSIGYDDTDES & 300 \\
\hline PLIN2-W & HCAEHIESRTLAIARNLTQQLQTTCHTLVSS Q QGLPQNIHDQANHLGVMAGDIYSVFHNA & 360 \\
\hline PLIN2-L & HAAEHIESRTLAIARNLTQQLQTTCHTLVANAGLPQNIHDQANHLGVMAGDIYSVFHNA & 360 \\
\hline PLIN2-W & SSFKEMSDGLLSSSKGQLQKMKESLDDVMDYLVNNTPLNWLVGPFYPQLTESQDAQSRGA & 420 \\
\hline PLIN2-L & SSFKEMSDGLLSSSKGQLQKMKESLDDVMDYLVNNTPLNWLVGPFYPQLTESQDAQSRGA & 420 \\
\hline PLIN & ENTTSPETQQPETH HIKPAPASSAW & \\
\hline PLIN & ENTTSPETQQPETh RIKPAPASSAWGSQSGDTSCTVATC 459 & \\
\hline
\end{tabular}

Figure 3: Comparison of amino acids sequences between Wujin and Landrace pigs Sequence indicates Landrace pigs and Sus was Wujin pigs
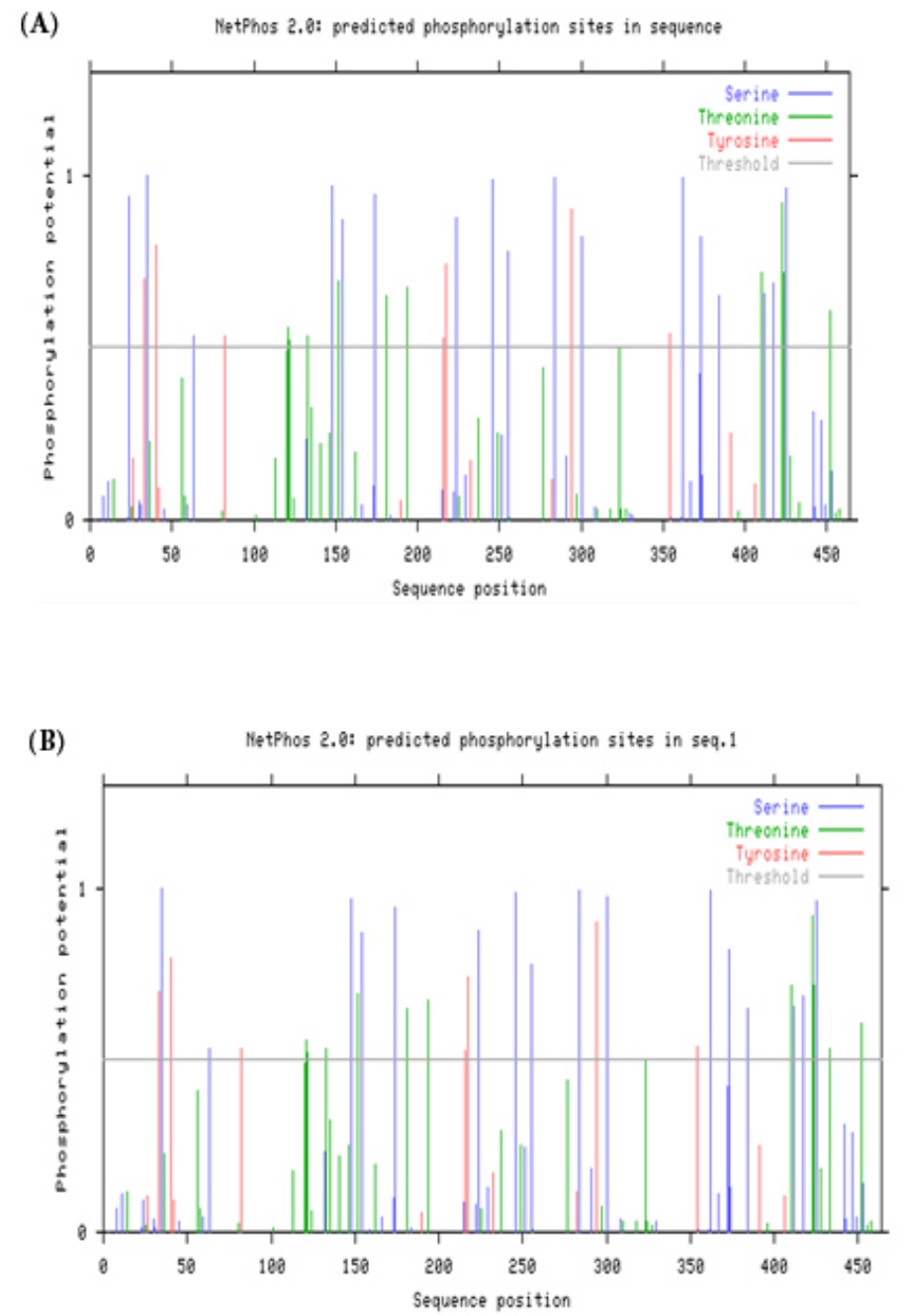

Figure 4: Predicted phosphorylation sites of deduced amino acids of PLIN2 A and B represent Wujin and Landrace pigs 


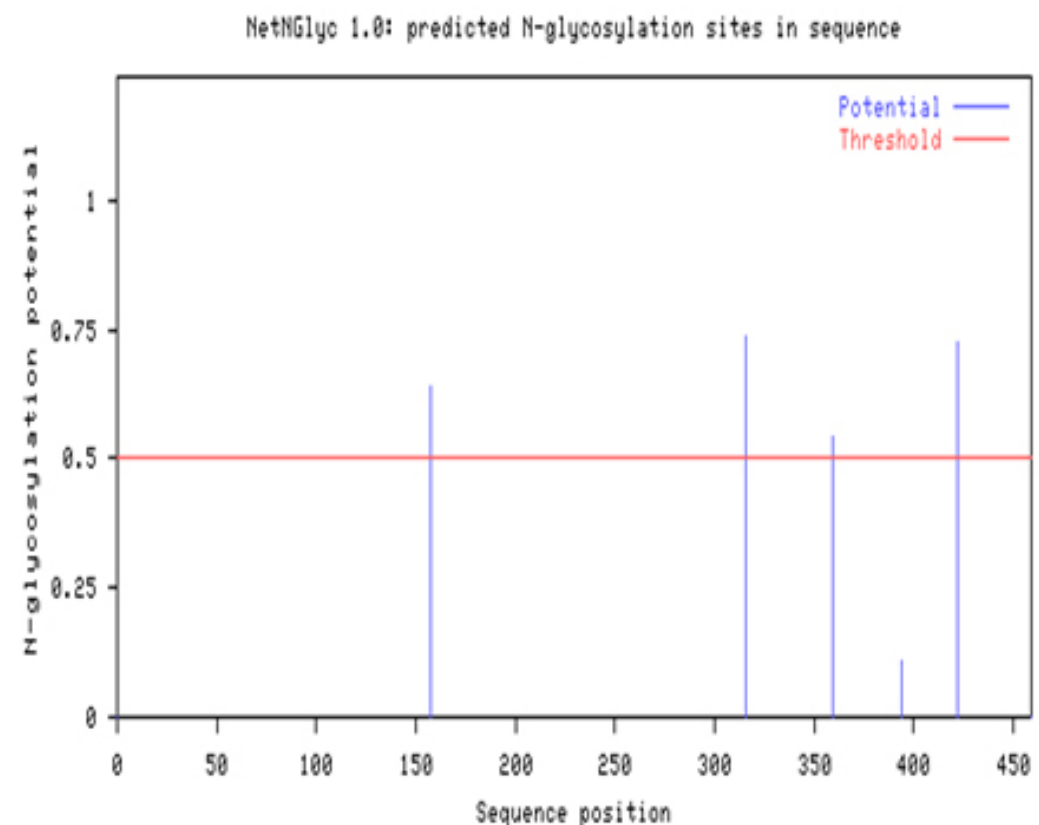

Figure 5: N-Glycosylation sites Prediction of PLIN2 deducted amino acids sequence

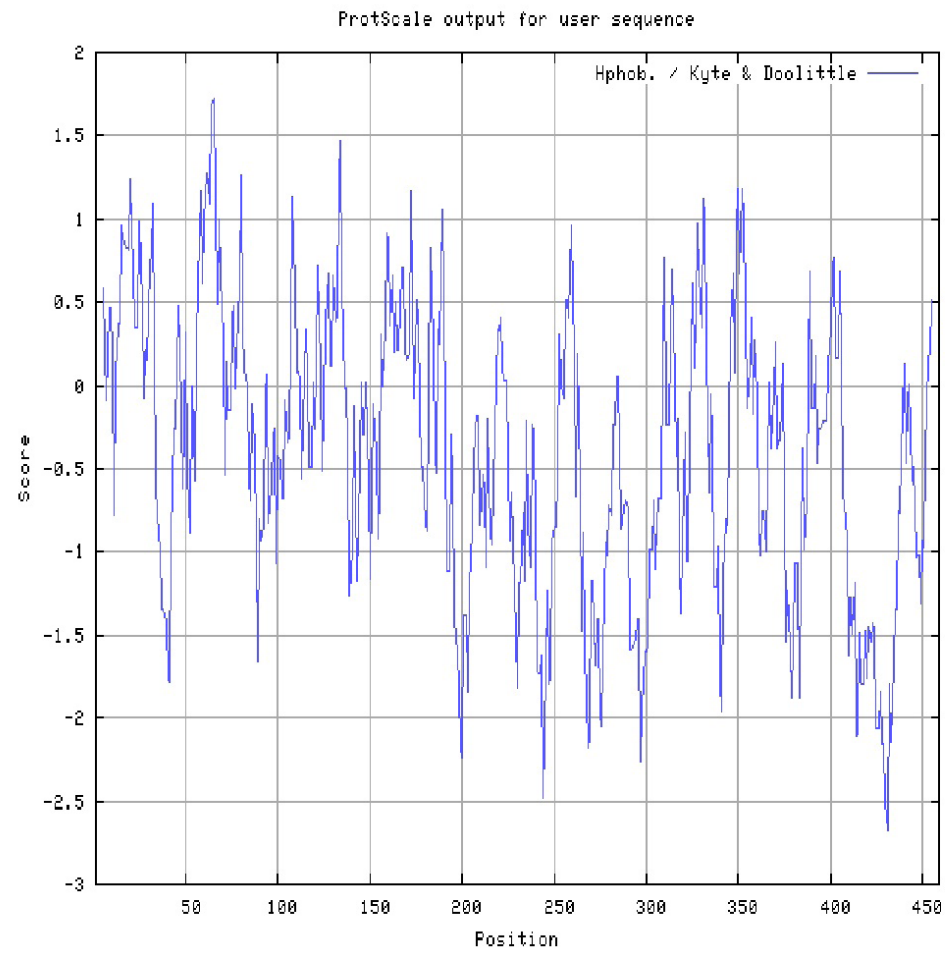

Figure 6: Hydrophobic characteristic analysis of PLIN2 deduced amino acids

\section{Swiss-Model homology modeling}

One model of porcine PLIN2 from Wujin and Landrace pigs was selected (E-value: 0.00e-1, QMEAN Z-Score: -2.23, QMEAN4: 0.63). Porcine PLIN2 protein was modeled by Mannose-6-phosphate receptor binding protein 1 (Tip47), C-terminal domain (ID: 1 sziA) as template. PLIN2 modeled residue range from 189 to 411 , which has $33.62 \%$ sequence identity with template sequence. Homology modeling indicated that the PLIN2 C terminus forms independently folding foue a-helical structure (Figure 7).

\section{Expression Level of PLIN2 mRNA}

Relative expression level of PLIN2 gene was detected using real-time PCR method. Figure 8 shows that the expression levels of PLIN2 gene in the Wujin pigs were significantly higher than Landrace pigs. 


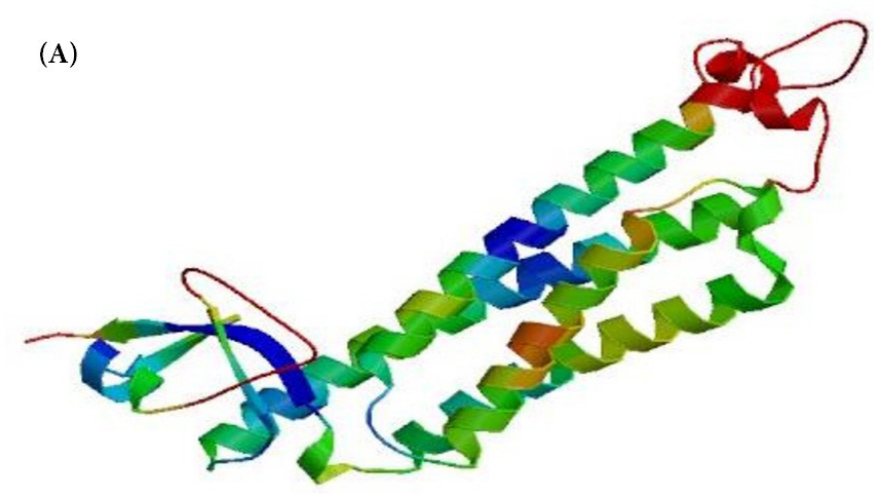

(B)

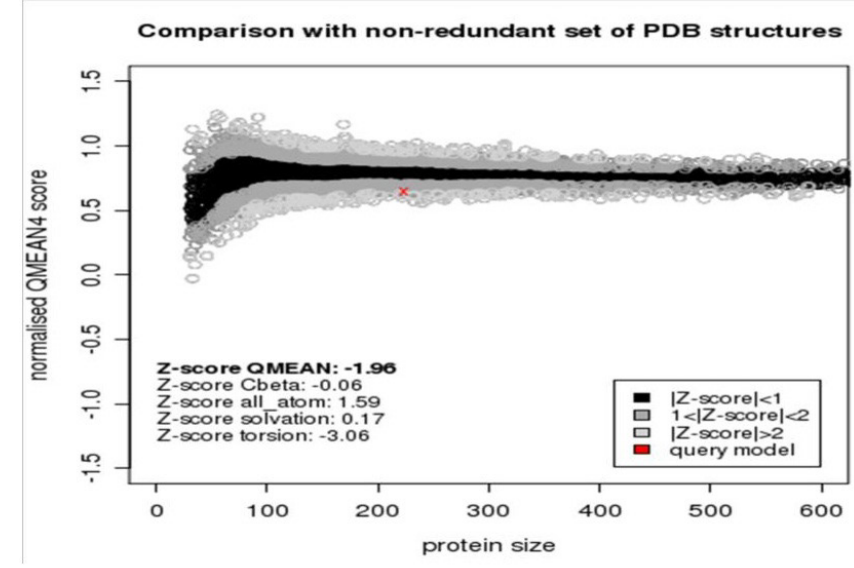

Figure 7: Swiss-model of PLIN2 deduced amino acids sequence A was cartoon model. B was estimated absolute quality of models

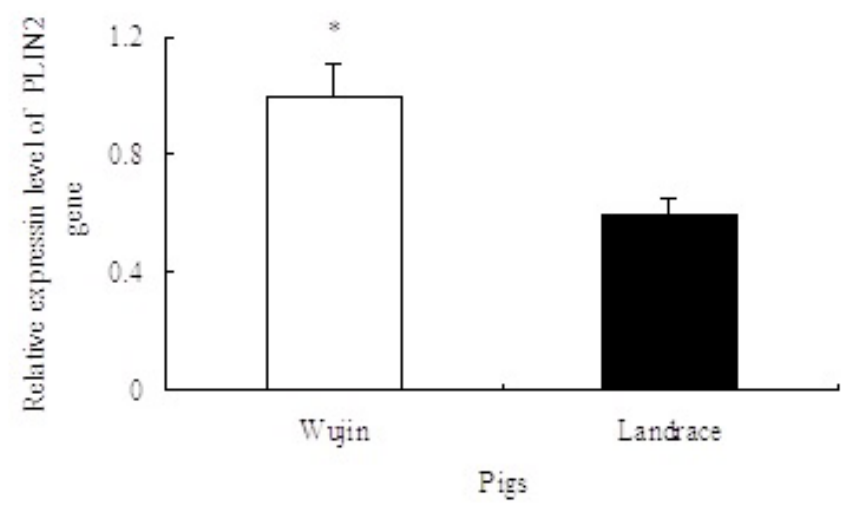

Figure 8: Relative expression level of PLIN2 gene

${ }^{*}$ represents significant difference, $\mathrm{P}<0.05, \mathrm{~N}=10$

\section{Discussion}

Several protein components associated with the surface of LDs have been identified. They are loosely grouped as a small protein family sharing a common N-terminal motif, called the PAT ((Perilipin, Adipophilin and Tip47) domain [20]. PLIN2 is one of them, which is a 50-kDa protein expressed in the majority of tissues [21], involved in the uptake of long chain fatty acids [22] and in the formation and stabilization of lipid droplets [23]. PLIN2 plays a role in protecting the neutral lipid stores from the activity of cytosolic lipases [24], and therefore in regulating lipid storage, in particular in non-adipose tissues. In fact, in adipose tissue PLIN2 is gradually replaced by PLIN1 during adipocyte maturation, whereas in other tissues, as skeletal muscle, PLIN2 remains the main lipid droplet associated protein [15]. 
The study showed that analysis of PLIN2 nucleotide and amino acid sequences revealed a high degree of identity between the Wujin and Landrace breeds, although base changes resulted in amino acid substitutions in the translated proteins. The amino acid substitutions led to the differential molecular weight and theoretical pI value as well as the predicted phosphorylation sites. Heid et al. [25] reported that the open reading frame of adipophilin encodes a polypeptide with a calculated molecular weight of 48.1 $\mathrm{kDa}$ and an isoelectric point of 6.72. Further analysis showed that the $\mathrm{N}$-glycosylated sites and hydrophilic characteristic as well as the SWISS-MODEL of PLIN2 protein were kept the same between Wujin and Landrace pigs. The function of PLIN2 protein was decided by the conformation of the protein structure [26]. Thus, this data suggested that the mutation of nucleic acids and amino acids sequences could not affect the function of PLIN2 to form and stabilize the lipid droplets. Moreover, the difference of IMF content between Wujin and Landrace pigs could not result from the mutation of nucleotide and animo acids sequence of PLIN2.

Swiss-Model is classical online modeling software, and widely used for modeling unknown protein sequence by homology. RasMol was used for visualizing the PDB files generated by Swiss-model in cartoon or three-dimensional (3-D) format. PLIN2 protein were Modeled by Swiss-Model, only high quality models with low E-value, low absolute value Z-Score, high QMEAN4 value were selected [19]. Homology modeling indicated that the ADPH C terminus forms a four-helix bundle motif. This structure can form stable membrane bilayer interactions [27]. PLIN2 C terminus is an independently folding $\alpha$-helical structure that is relatively resistant to urea denaturation [27]. Liposome binding studies showed that the purified $\mathrm{C}$ terminus binds to phospholipid membranes through electrostatic dependent interactions, and cell culture studies documented that it localizes to the plasma membrane [27]. The present study showed PLIN2 belong to the hydrophilic protein. PLIN2 C terminus forms a stable membrane binding helical structure that is important for lipid droplets secretion.

Skeletal muscle lipid droplet-associated proteins (PLINs) are thought to regulate lipolysis through protein-protein interactions on the lipid droplet surface [28]. Non-ubiquitous PLIN2 expression in pigs has been recently reported by Tao et al. [29], which indicated that PLIN2 gene did not express in muscle tissue. Davoli et al. [12] also detected PLIN2 gene expression analysis in skeletal muscle of Italian Large White and Italian Duroc pigs divergent for backfat thickness and visible intermuscular fat showed a trend of higher expression level in pigs with higher intermuscular fat. In the present study, PLIN2 expressed in the muscle tissue and its expression level in Wujin pigs was significantly higher than Landrace pigs. Meanwhile, IMF content in Wujin pigs is significantly higher compared with Landrace pigs [8]. PLIN2 localized within myofibers with high lipid content [16]. PLIN2 expression was higher in higher IMF muscles [12]. Therefore, this data suggested that the expression level of PLIN2 gene would contribute to the IMF content.

In conclusion, the mutation of nucleic acids and amino acids between Wujin and Landrace could not affect the structure of PLIN2. However, the difference of expression level between two pig breeds would be responsible for the IMF content variants.

\section{Acknowledge}

This work was supported by Yunnan Natural Science Foundation, China (2009CD056), National Natural Science Foundation of China (No 31060331 and 31260592), National Key Program of Transgenic Project of China (No. 2009ZX08009-140B), Major Projects of Yunnan Provincial Educational Department, China (No. ZD2015007) and Creative Projects of Yunnan Provincial Key Laboratory of Animal Nutrition and Feed Science, China(No. DYCX2015002).

\section{References}

1. Fernandez X, Monin G, Talmant A, Mourot J, Lebret B (1999) Influence of intramuscular fat content on the quality of pig meat-1. Composition of the lipid fraction and sensory characteristics of $\mathrm{m}$. longissimus lumborum. Meat Sci 53: 59-65.

2. Sellier P (1998) Genetics of meat and carcass traits. In: The genetics of the pig. Rothschild MF, Ruvinsky A (Eds) CAB International, Wallingford, UK, 463.

3. Kinyamu HK, Ewan RC (1994) Energy and protein metabolism of the Chinese pig. J Anim Sci 72: 2068-74.

4. Yen JT, Nienaber JA, Klindt J, Crouse JD. (1991) Effect of ractopamine on growth, carcass traits, and fasting heat production of US contemporary crossbred and Chinese Meishan pure- and crossbred pigs. J Anim Sci 69: 4810-7.

5. Young LD (1992) Comparison of Meishan, Fengjing, Minzhu and Duroc swine: effects on postweaning growth, feed efficiency, and carcass traits. J Anim Sci 70: 2020-9.

6. Zhang Xi, Zhao Su-mei, GE Chang-rong, LAI Hua, LI Chang-qiang, et al. (2008) Effects of dietary composition and digestive energy levels on meat quality in Wujin Pigs. Chin J Anim Nutri 20: 58-65.

7. GE Chang-rong, Zhao Su-mei, Zhang Xi, Lai Hua, Li Chang-qiang, et al. (2008) Effects of dietary protein levels on meat quality in Wujin Pigs. Acta Veter Zoote Sini 39: 1692-1700.

8. Zhao SM, Ren LJ, Chen L, Zhang X, Cheng ML, et al. (2009) Differential expression of lipid metabolism related genes in porcine muscle tissue leading to different intramuscular fat deposition. Lipids 44: 1029-37.

9. Gao SZ, Zhao SM (2009) Physiology, affecting factors and strategies for control of pig meat intramuscular fat. Recent Pat Food Nutr Agric 1: 59-74

10. Kim TH, Choi BH, Chang GW, Lee KT, Lee HY, et al. (2005) Molecular characterization and chromosomal mapping of porcine adipose differentiation-related protein (ADRP). J Anim Breed Genet 122: 240-6.

11. Nie T, Zhao XL, Qiu H, Xia T, Chen XD, et al. (2005) Sequence analysis and map assignment of pig SREBF2 and ADFP. Anim Genet 36: 455-7.

12. Davoli R, Gandolfi G, Braglia S, Comella M, Zambonelli P, et al. (2011) New SNP of the porcine Perilipin 2 (PLIN2) gene, association with carcass traits and expression analysis in skeletal muscle. Mol Biol Rep 38: 1575-83. 
13. Liu G, Jennen DG, Tholen E, Juengst H, Kleinwächter T, et al. (2007) A genome scan reveals QTL for growth, fatness, leanness and meat quality in a DurocPietrain resource population. Anim Genet 38: 241-52.

14. Thomsen H, Lee HK, Rothschild MF, Malek M, Dekkers JC (2004) Characterization of quantitative trait loci for growth and meat quality in a cross between commercial breeds of swine. J Anim Sci 82: 2213-28.

15. P. Beeckmann, J. Schröffel Jr, G. Moser, H. Bartenschlager, G. Reiner, et al. (2003) Linkage and QTL mapping for Sus scrofa chromosome 1. J Anim Breed Genet 120: $1-10$.

16. Gandolfi G, Mazzoni M, Zambonelli P, Lalatta-Costerbosa G, Tronca A, et al. (2011) Perlipin 1 and perlipin 2 protein localization and gene expression study in skeletal muscles of European cross-breed pigs with different intramuscular fat contents. Meat Sci 88: 631-7.

17. Blom N, Gammeltoft S, Brunak S (1999) Sequence and structure-based prediction of eukaryotic protein phosphorylation sites. J Mol Biol 294 : 1351-62.

18. Gupta R, Jung E, Brunak S (2004) Prediction of N-glycosylation sites in human proteins. In preparation.

19. Kiefer F, Arnold K, Künzli M, Bordoli L, Schwede T (2009) The SWISS-MODEL Repository and associated resources. Nucleic Acids Res 37: D387-92.

20. Perry EB, John TT, Michael WA, (2009) PAT proteins, an ancient family of lipid droplet proteins that regulate cellular lipid stores. Biochim Biophys Acta 1791: 419-40.

21. Brasaemle DL, Barber T, Wolins NE, Serrero G, Blanchette-Mackie EJ, et al. (1997) Adipose differentiation-related protein is an ubiquitously expressed lipid storage droplet-associated protein. J Lipid Res 38: 2249-63.

22. Gao J, Serrero G (1999) Adipose differentiation related protein (ADRP) expressed in transfected COS-7 cells selectively stimulates long chain fatty acid uptake. J Biol Chem 274: 16825-30.

23. Imamura M, Inoguchi T, Ikuyama S, Taniguchi S, Kobayashi K, et al. (2002) ADRP stimulates lipid accumulation and lipid droplet formation in murine fibroblasts. Am J Physiol Endocrinol Metab 283: E775-83.

24. Listenberger LL, Ostermeyer-Fay AG, Goldberg EB, et al. (2007) Adipocyte differentiation-related protein reduces the lipid droplet association of adipose triglyceride lipase and slows triacylglycerol turnover. J Lipid Res 48: 2751-61.

25. Heid HW, Moll R, Schwetlick I, Rackwitz HR, Keenan TW (1998) Adipophilin is a specific marker of lipid accumulation in diverse cell types and diseases. Cell Tissue Res 294: 309-21.

26. Wu J, Jiao ZY, Lu HL, Zhang J, Lin HH, et al. (2011) The molecular mechanism of acylation stimulating protein regulation of adipophilin and perilipin expression: involvement of phosphoinositide 3-kinase and phospholipase C. J Cell Biochem 112: 1622-9.

27. Chong BM, Russell TD, Schaack J, Orlicky DJ, Reigan P, et al. (2011) The adipophilin C terminus is a self-folding membrane-binding domain that is important for milk lipid secretion. J Biol Chem 286: 23254-65.

28. MacPherson RE, Herbst EA, Reynolds EJ, Vandenboom R, Roy BD, et al. (2012) Subcellular localization of skeletal muscle lipid droplets and PLIN family proteins OXPAT and ADRP at rest and following contraction in rat soleus muscle. Am J Physiol Regul Integr Comp Physiol 302: R29-36.

29. Tao X, Jihong Y, Li G, Bin F, Yi Z, et al. (2008) Cloning, chromosome mapping and expression pattern of porcine PLIN and M6PRBP1 genes. Genet Sel Evol 40: $215-26$. 